\title{
Principales Tendencias Iberoamericanas en Psicología Clínica. Un Estudio Basado en la Evidencia Científica
}

\author{
Main Trends in Iberoamerican Clinical Psychology. A Study Based in Scientific Evidence.
}

\author{
Sebastián Lillo ${ }^{1}$ \\ Universidad de Santiago de Chile, Chile \\ Natalia Martini² \\ Universidad de Chile, Chile
}

(Rec: 20 de junio de 2013 / Acept: 1 de octubre de 2013)

\begin{abstract}
Resumen
La disponibilidad de técnicas que permiten el análisis de gran cantidad de datos, entrega la posibilidad de realizar estudios que permiten caracterizar el desarrollo y la evolución de la ciencia psicológica a través de parámetros objetivos, entregando perfiles de las principales tendencias. Se presenta un enfoque hibrido basado en análisis cientométrico y una interpretación de comunidades bibliográficas basadas en la proporción de referencias compartidas (Grawin, 2012) de artículos iberoamericanos en la base de datos Web of Knowledge. Los resultados arrojan una concentración de la investigación en Depresión (9\%) y DemenciaAlzheimer ( $8 \%$ ), así como una fuerte orientación cuantitativa a través de la validación y uso de instrumentos psicométricos. Mientras España produce 54\% de la ciencia y lidera 44\% de la producción más influyente (citada), Latinoamérica produce 37\% y lidera 13\% (Brasil 10\%, Chile 1\%, Argentina 1\%, Colombia 1\%). Se discuten las implicancias de estos resultados, así como las consecuencias y futuras aplicaciones de estas herramientas para practicantes e investigadores.

Palabras clave: psicología clínica, análisis cientométrico, Iberoamérica
\end{abstract}

\begin{abstract}
The availability of techniques that allows the analysis of large quantities of data permits the construction of studies that can characterize the development and evolution of the psychological science through objective parameters, delivering profiles of the main trends. In this article we present a hybrid approach, based on a scientometric analysis and an interpretation of bibliographic communities based on the proportion of shared references (Grawin, 2012) of iberoamerican articles within the Web of Knowledge database. The results show a strong concentration of research on Depression (9\%) and Alzheimer (8\%), as a heavy use of quantitative and psychometric approaches. While Spain produces $54 \%$ of the science and leads $44 \%$ of the most influential (cited) production, Latin America produces $37 \%$ and leads $13 \%$ (Brasil 10\%, Chile 1\%, Argentina 1\%, Colombia 1\%). The repercussions of the results are discussed, as the consequences and future applications of these tools for practitioners and researchers.

Key words: clinical psychology, scientometric analysis, Ibero-America
\end{abstract}

\section{Introducción}

La psicología como disciplina científica se desarrolla a través de la publicación de los conocimientos o investigaciones realizadas. Sin embargo, de la mano de esta necesidad surge la dificultad para medir los niveles de productividad de los investigadores, la que se inicia con la definición de productividad en sí misma y deriva en sus formas de medición (Duffy, Jadidian, Webster y Sandell, 2011).

Uno de los caminos para aproximarse al estudio de la productividad científica en el área es la cientometría, estudio cuantitativo de la ciencia como problema multidimensional,

Agradecimientos:

${ }^{1}$ El autor desea agradecer al proyecto USA 1204 del Ministerio de Educación/CITIAPS

${ }^{2}$ La autora desea agradecer a CONICYT PCHA/Magíster Nacional/2013 - folio 22130154.

Correspondencia: Sebastián Lillo Vidal, sebastian.lillo@usach.cl, Vicerrectoría de Investigación, Desarrollo e Innovación, Universidad de Santiago de Chile. Alameda 3363, Casa Central. 
incluyendo los investigadores y los textos como objeto de estudio. Como tal, comprende el crecimiento de la ciencia, el desarrollo de disciplinas y subdisciplinas, la creatividad y productividad de los científicos y la relación entre la ciencia y el desarrollo económico, entre otros. (Amézquita, Martínez, Martínez y Maza, 2011)

Se diferencia de otras aproximaciones de estudio de la ciencia como la bibliometría o la infometría, en tanto que la cientometría comprende el estudio de disciplinas y campos científicos, más que el artículos, libros, autores o palabras y bases de datos respectivamente (Amézquita, Martínez, Martínez y Maza, 2011). Es decir, la cientometría comprende un análisis más global de la ciencia, buscando un nivel más comprensivo, relacionándola también con otros indicadores de desarrollo como la economía o política.

Las investigaciones relacionadas al estudio de la productividad de la psicología clínica en países de habla hispana de los últimos años hacen referencia a análisis de las revistas más importantes dentro del área (Ariza y Reina Granados, 2012; Quevedo-Blasco y López-López, 2011), el nivel de desarrollo en cada país identificando aquellos de mayor impacto (Vera-Villarroel, López-López, Lillo y Silva, 2011), la influencia del sexo de los investigadores en el nivel de producción, a nivel general de la psicología (Barrios, Vilarroya, y Borrego 2013), internacionalización de las revistas iberoamericanas (Zych y Buela-Casal, 2010) y análisis bibliométrico por países de la producción en revistas de psicología (Navarrete-Cortés, Fernández-López, LópezBaena, Quevedo-Blasco y Buela-Casal, 2010), entre otros.

En el presente estudio esta se busca dar cuenta de las tendencias de investigación de los últimos 5 años en Iberoamérica, contemplando en el análisis las temáticas con mayor impacto dentro de la psicología clínica, los países con mayor presencia en la investigación abordando así los diferentes temas investigados dentro del área los últimos años.

Así, en esta investigación se abordará, a través de un análisis basado en la cientometría, la producción científica en psicología clínica en Iberoamérica en los últimos 5 años, siendo una de las tres áreas de la psicología con mayor cantidad de investigaciones actualmente (Barrios, Vilarroya y Borrego, 2013).

\section{Método}

\section{Unidad de Análisis}

Los datos para esta investigación fueron extraídos desde la Web of Knowledge (ex ISI) que contiene más de
54 millones de artículos indexados en todas las áreas del conocimiento (Reuters, 2013) La búsqueda se refino por categorías de la ciencia, años y países latinoamericanos.

\section{Procedimiento}

Se descargaron todos los artículos ${ }^{1}$ en la categoría (WC) Psicología Clínica para el período 2008-2012, para los países iberoamericanos. La definición operacional de ciencia Iberoamericana corresponde a todos aquellos artículos donde participa al menos, un científico con afiliación a alguna institución Iberoamericana. Esto dio lugar a una base de 1421 artículos que fueron utilizados en todos los análisis posteriores. La determinación del líder de cada artículo se asignó al Autor para Correspondencia o "Corresponding Author" asumiendo el lugar especial que ocupa este dentro del equipo de investigación (MoyaAnegón, Guerrero-Bote, Bornmann y Moed, 2013). Este enfoque ha sido exitosamente utilizado posteriormente en la elaboración del ranking SJR (SCIMago Group, 2013). El sexo del autor fue clasificado manualmente por los autores en base al nombre designado como de correspondencia (columna CA de la base de datos).

El análisis de Grafos posterior se realizó en baso al trabajo de Grawin $(2011,2012)$. Para la investigación en los últimos cinco años se realizo un análisis de comunidades bibliográficas. Esto es, se analizaron las referencias de cada artículo y se organizaron en "comunidades". Este enfoque, proveniente desde las ciencias de los sistemas complejos permite utilizar un enfoque objetivo en base de caracteristicas de las publicaciones y sus conecciones para detectar principios universales en la ciencia, como contraparte al clásico análisis cualitativo basado en juicio de expertos de renombre (Grawin, 2011).

Las comunidades son definidas de acuerdo a cuantas referencias "comparten" dos artículos determinados. Si el número supera un límite determinado, los artículos componen una comunidad. Cada comunidad tiene un mínimo de 10 artículos. Las referencias son pesadas de acuerdo a la similaridad de cosenos propuesta por Kessler y la detección de comunidades de acuerdo al algoritmo de

\footnotetext{
$(\mathrm{WC}=(\mathrm{PSYCHOLOGY} \mathrm{CLINICAL}) \mathrm{AND}$ PY $=(2012$ OR 2011 OR 2010 or 2009 or 2008)) AND Document Types=(Article) Refined by: Countries/Territories $=($ SPAIN OR BRAZIL OR PORTUGAL OR CHILE OR MEXICO OR ARGENTINA OR COLOMBIA OR PERU OR CUBA OR VENEZUELA OR COSTA RICA OR DOMINICAN REP OR URUGUAY OR NICARAGUA ) Databases=SCI-EXPANDED, AyHCI, SSCI.
} 
Louvain (Figura1) que busca maximizar las relaciones intra comunidad y minimizar las relaciones inter comunidades. Los cálculos fueron realizados con el software Bibliotools diseñado por Grawin (2011).

El manejo de la base de datos fue realizado en MS Excel 2010. Los análisis estadísticos fueron realizados en SPSS 20. Para la visualización de los grafos se utilizó el software Gephi 0.8 .

$$
\omega i j=\frac{|R i \cap R j|}{\sqrt{\mid R i \cap R j}}
$$

$$
Q \omega=1 / 2 \Omega \sum_{i j}\left[w_{i j}-\frac{w i w j}{2 \Omega}\right] \delta(\mathrm{ci}, \mathrm{cj})
$$

Figura 1. Similiaridad de Cosenos y Algoritmo de Louvain

\section{Resultados}

Como modo de caracterizar la producción científica de la disciplina, se muestran diferentes descriptivos con respecto al comportamiento de la producción entre los años 2008-2012.

En esta tabla 1, se observan los principales descriptivos que caracterizan la producción, reflejando los diez primeros resultados en cada categoría, ordenados por número de apariciones. Por ejemplo, en relación a instituciones, la Universidad de Barcelona aparece mencionada 113 veces en la base de datos, convirtiéndola en la universidad con mayor presencia en la misma. Por otro lado, las dos primeras referencias más utilizadas corresponden a las versiones de 1994 y 2000 del Manual Diagnóstico Estadístico de Desórdenes Mentales.

En la figura 2 se puede observar la evolución de la producción científica en psicología clínica. Esta ha aumentado de manera coherente con la tendencia de crecimiento de todas las ciencias (Olesen y von Ins, 2010).

\begin{tabular}{|c|c|c|c|c|c|}
\hline Institución & Apariciones & Categoría (WC) & Apariciones & Autores & Apariciones \\
\hline UNIV. BARCELONA & 113 & Psychology, Clinical & 1348 & Fernández-Aranda F & 22 \\
\hline UNIV. GRANADA & 94 & Psychology & 423 & Jiménez-Murcia S & 21 \\
\hline UNIV. AUTÓNOMA BARCELONA & 82 & Psychiatry & 382 & Penelo E & 21 \\
\hline UNIV. SAO PÃULO & 67 & Clinical Neurology & 102 & Vieta E & 19 \\
\hline UNIV. VALENCIA & 60 & Gerontology & 70 & Echeburua E & 18 \\
\hline CIBERSAM & 50 & Geriatrics y Gerontology & 70 & Granero R & 18 \\
\hline UNIV. AUTONÓMA MADRID & 42 & Substance Abuse & 50 & Menchon JM & 16 \\
\hline UNIV. COIMBRA & 40 & Social Sciences, Interdisciplinary & 46 & Irurtia MJ & 15 \\
\hline UNIV. MINHO & 38 & Neurosciences & 44 & Yassuda MS & 14 \\
\hline UNIV. BASQUE COUNTRY & 37 & Family Studies & 40 & Caballo VE & 14 \\
\hline
\end{tabular}

Tabla 1. Principales Descriptivos de la Producción iberoamericana en Psicología Clínica.

\begin{tabular}{|c|c|c|c|c|c|}
\hline Palabra Clave & Apariciones & Journal & Apariciones & Referencias & Apariciones \\
\hline DEPRESSION & 130 & BEHAV PSYCHOL & 136 & $\begin{array}{l}\text { American psychiatric ASSOCIATION, 1994, DIAGN } \\
\text { STAT MAN MENT, 0, } 0\end{array}$ & 142 \\
\hline PREVALENCE & 113 & INT J CLIN HLTH PSYC & 116 & $\begin{array}{l}\text { American psychiatric ASSOCIATION, 2000, DIAGN } \\
\text { STAT MAN MENT, } 0,0\end{array}$ & 117 \\
\hline SYMPTOMS & 82 & REV ARGENT CLIN PSIC & 96 & Montero I, 2007, INT J CLIN HLTH PSYC, 7, 847 & 94 \\
\hline WOMEN & 80 & REV IBEROAM DIAGN EV & 85 & Cohen J, 1988, STAT POWER ANAL BEHA, 0, 0 & 75 \\
\hline VALIDATION & 79 & TER PSICOL & 82 & Folstein MF, 1975, J PSYCHIAT RES, 12, 189 & 52 \\
\hline VALIDITY & 79 & INT PSYCHOGERIATR & 70 & $\begin{array}{l}\text { Ramos-Álvarez MM, 2008, INT J CLIN HLTH } \\
\text { PSYC, } 8,751\end{array}$ & 47 \\
\hline SCALE & 74 & J CLIN PSYCHIAT & 61 & Beck AT, 1961, ARCH GEN PSYCHIAT, 4, 561 & 42 \\
\hline BEHAVIOR & 71 & PSYCHOL MED & 56 & Hu LT, 1999, STRUCT EQU MODELING, 6, 1 & 37 \\
\hline ADOLESCENTS & 70 & EUR EAT DISORD REV & 37 & Mckhann G, 1984, NEUROLOGY, 34, 939 & 37 \\
\hline DISORDERS & 70 & ADDICT BEHAV & 32 & Carretero-Dios H, 2007, INT J CLIN HLTH PSYC, 7, 863 & 36 \\
\hline
\end{tabular}




\section{Producción Iberoamericana WoK-ISI}

Psicología Clínica 2008-2012

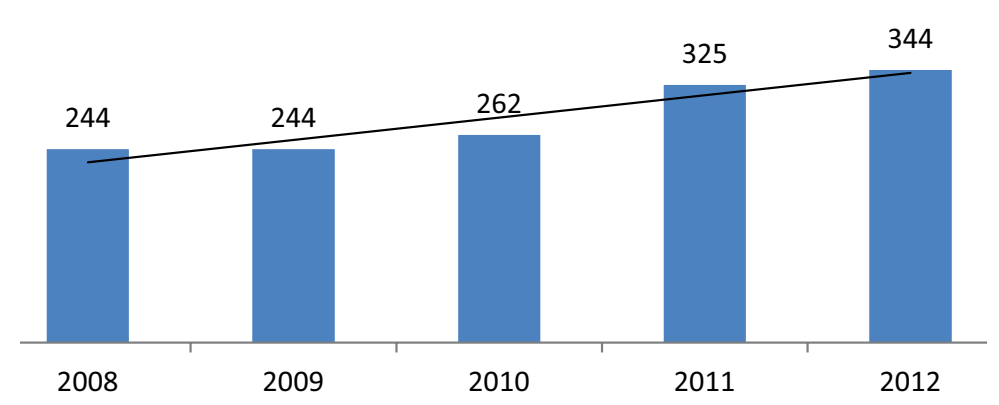

Figura 2. Producción iberoamericana por año.

\section{Producción Iberoamericana WoK ISI}

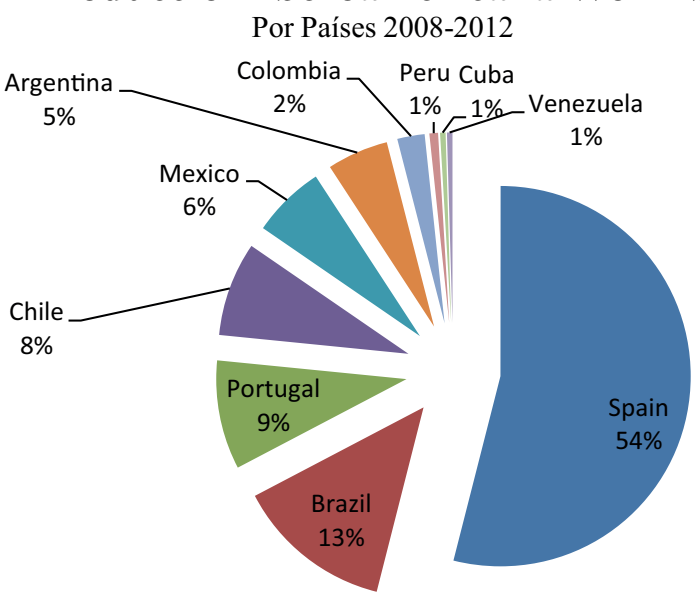

Figura 3. Producción total iberoamericana por países.

En la figura 3 se puede observar la producción iberoamericana por países. Destaca la productividad de España, que domina el panorama con un 54\% de la productividad total, seguido de Brasil, Portugal y Chile.

El análisis del liderazgo de los 10\% de los artículos más influyentes de acuerdo a las citas recibidas (Figura 4) muestra un escenario distinto. España, Brasil y Portugal retienen su influencia, pero los países latinoamericanos Chile, Argentina, Colombia y México bajan a porcentajes cercanos al $1 \%$.

Del total de artículos un 70\% está escrito en inglés y un $28 \%$ en español mostrando un predominio de la lengua inglesa como la lengua franca de la ciencia, incluso para los autores iberoamericanos. El 2\% restante se encuentra en portugués, a pesar de que Brasil y Portugal representan casi el $22 \%$ de las publicaciones totales.
Producción Iberoamericana más influyente

Liderazgo en $10 \%$ de artículos más citados

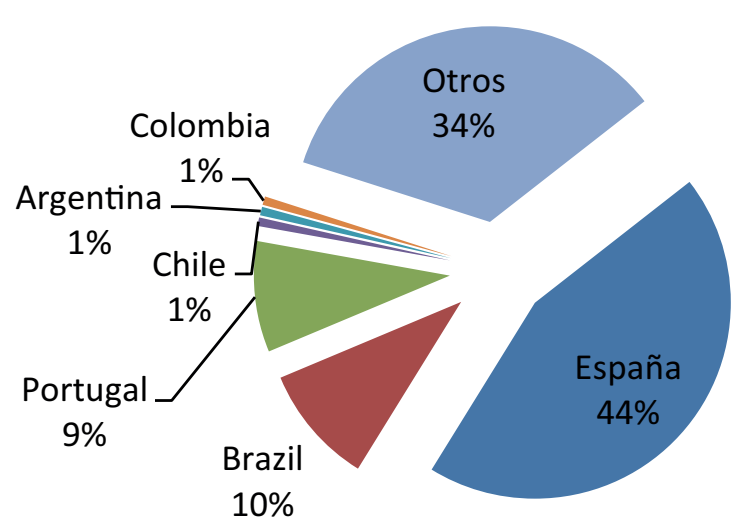

Figura 4. Análisis del 10\% de la producción iberoamericana mas citada por países

En la tabla 2 se presentan los diez artículos con mayor cantidad de citaciones en el periodo, representando la ciencia más influyente y la que ha tenido más impacto en la comunidad científica. El análisis de estos artículos revela ciertas tendencias. Ninguno de estos artículos se publicó en una revista iberoamericana. La mayoría de los artículos (6 de 10) fue liderado por investigadores no iberoamericanos, y destaca que ningún latinoamericano lideró alguno de estos artículos (el primer latinoamericano en liderar un artículo influyente aparece recién en el puesto número 17).

En cuanto a los temas tratados en los artículos estudiados, el más citado habla sobre las opiniones de expertos en relación a comorbilidad entre enfermedades somáticas y psiquiátricas, estableciendo una relación entre la psiquiatría y otras áreas de la medicina. 
Tabla 2. 10 Artículos más citados en el periodo con autores iberoamericanos.

\begin{tabular}{|c|c|c|}
\hline Título & Revista & Autor líder \\
\hline $\begin{array}{l}\text { Comorbid somatic illnesses in patients with severe mental } \\
\text { disorders: Clinical, policy, and research challenges }\end{array}$ & $\begin{array}{l}\text { JOURNAL OF CLINICAL } \\
\text { PSYCHIATRY }\end{array}$ & $\begin{array}{l}\text { Fleischhacker, WW } \\
\text { Med. Univ. Innsbruck, } \\
\text { Austria. }\end{array}$ \\
\hline $\begin{array}{l}\text { Failure to deactivate in the prefrontal cortex in schizophrenia: } \\
\text { dysfunction of the default mode network? }\end{array}$ & $\begin{array}{l}\text { PSYCHOLOGICAL } \\
\text { MEDICINE }\end{array}$ & $\begin{array}{l}\text { Pomarol-Clotet, } \\
\text { Benito Menni Complex, } \\
\text { Spain. }\end{array}$ \\
\hline $\begin{array}{l}\text { Efficacy and Safety of Lamotrigine as Add-On Treatment to } \\
\text { Lithium in Bipolar Depression: A Multicenter, Double-Blind, } \\
\text { Placebo-Controlled Trial }\end{array}$ & $\begin{array}{l}\text { JOURNAL OF CLINICAL } \\
\text { PSYCHIATRY }\end{array}$ & $\begin{array}{l}\text { Van der Loos, MLM } \\
\text { IsalaKlin, DeptPsychiat, } \\
\text { Netherlands. }\end{array}$ \\
\hline $\begin{array}{l}\text { Early adversity and 5-HTT/BDNF genes: new evidence of } \\
\text { gene-environment interactions on depressive symptoms in a } \\
\text { general population }\end{array}$ & $\begin{array}{l}\text { PSYCHOLOGICAL } \\
\text { MEDICINE }\end{array}$ & $\begin{array}{l}\text { Fananas, L } \\
\text { Univ. Barcelona, } \\
\text { Spain. }\end{array}$ \\
\hline $\begin{array}{l}\text { Construct validity of the Trail Making Test: Role of task- } \\
\text { switching, working memory, inhibition/interference control, } \\
\text { and visuomotor abilities }\end{array}$ & $\begin{array}{l}\text { JOURNAL OF THE } \\
\text { INTERNATIONAL } \\
\text { NEUROPSYCHOLOGICAL } \\
\text { SOCIETY }\end{array}$ & $\begin{array}{l}\text { Perianez, JA } \\
\text { Univ. Complutense, } \\
\text { Spain. }\end{array}$ \\
\hline $\begin{array}{l}\text { Efficacy of the Novel Antidepressant Agomelatine on the } \\
\text { Circadian Rest-Activity Cycle and Depressive and Anxiety } \\
\text { Symptoms in Patients With Major Depressive Disorder: A } \\
\text { Randomized, Double-Blind Comparison With Sertraline }\end{array}$ & $\begin{array}{l}\text { JOURNAL OF CLINICAL } \\
\text { PSYCHIATRY }\end{array}$ & $\begin{array}{l}\text { Kasper, } \mathrm{S} \\
\text { Med Univ. Vienn, } \\
\text { Austria. }\end{array}$ \\
\hline $\begin{array}{l}\text { Neurocognitive impairment in bipolar patients with and without } \\
\text { history of psychosis }\end{array}$ & $\begin{array}{l}\text { JOURNAL OF CLINICAL } \\
\text { PSYCHIATRY }\end{array}$ & $\begin{array}{l}\text { Vieta, E } \\
\text { Univ. Barcelona, } \\
\text { Spain. }\end{array}$ \\
\hline $\begin{array}{l}\text { Celecoxib as an adjunct in the treatment of depressive or mi- } \\
\text { xed episodes of bipolar disorder: a double-blind, randomized, } \\
\text { placebo-controlled study }\end{array}$ & $\begin{array}{c}\text { HUMAN } \\
\text { PSYCHOPHARMACOLOGY- } \\
\text { CLINICAL AND } \\
\text { EXPERIMENTAL }\end{array}$ & $\begin{array}{c}\text { Soares, JC } \\
\text { Univ. N Carolina, USA. }\end{array}$ \\
\hline $\begin{array}{l}\text { Using Icon Arrays to Communicate Medical Risks: Overcoming } \\
\text { Low Numeracy }\end{array}$ & HEALTH PSYCHOLOGY & $\begin{array}{c}\text { Galesic, } \mathrm{M} \\
\text { Max Planck Inst.,Germany }\end{array}$ \\
\hline $\begin{array}{l}\text { Delay and Reward Choice in ADHD: An Experimental Test of } \\
\text { the Role of Delay Aversion }\end{array}$ & NEUROPSYCHOLOGY & $\begin{array}{l}\text { Sonuga-Barke, EJS, } \\
\text { Univ. Southampton, England. }\end{array}$ \\
\hline
\end{tabular}

Destacan los estudios sobre bipolaridad y esquizofrenia, efectos de fármacos como lamotrigina, agomelatina, celecoxib y ziprazidona, estudios sobre procesos cognitivos en diferentes trastornos psiquiátricos y la influencia genética en la enfermedad mental.

Se aprecia una predominancia de estudios experimentales, pese a que el más citado (Fleischhacker et al., 2008) es un estudio de opinión de expertos y orientado a la psiquiatría.

\section{Comunidades Bibliográficas}

En la figura 6 es posible apreciar las comunidades que representan la mayor concentración de ciencia Iberoamericana en psicología clínica. Cada circulo representa una "comunidad" que comparte referencias bibliográficas. Cada una se encuentra más o menos relacionada con otras comunidades, de acuerdo al número de referencias que compartan. Como se observa en mayor detalle en la tabla 3, los instrumentos 


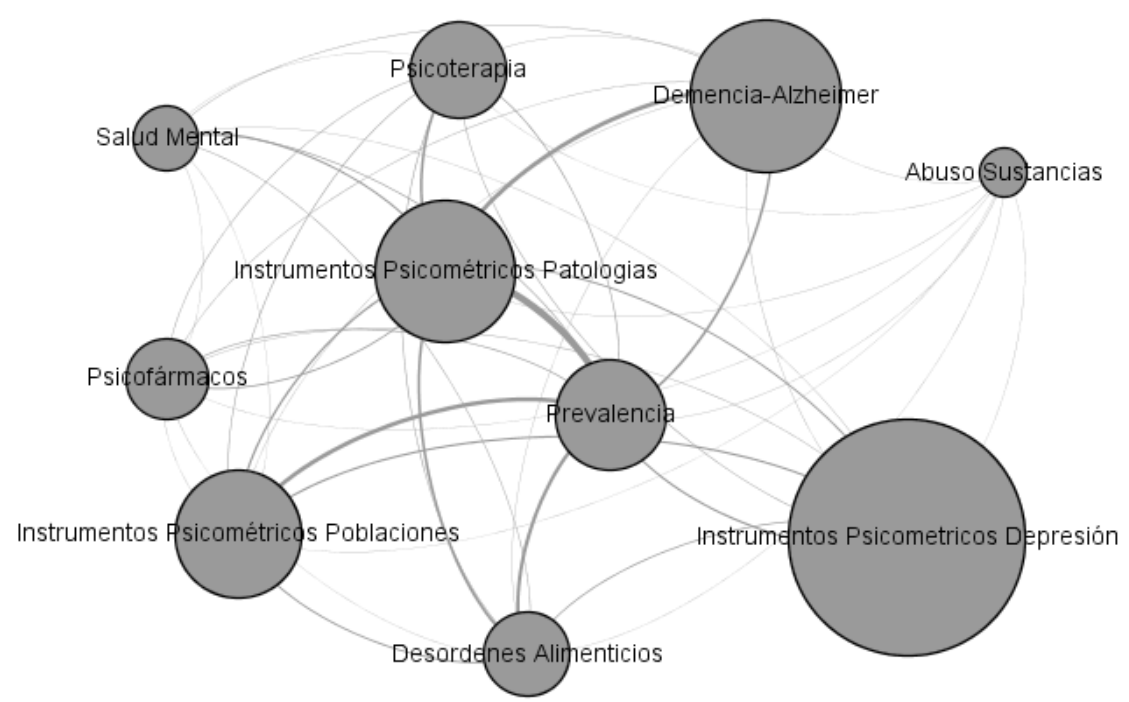

Figura 5. Gráfico de Comunidades Bibliográficas. Tamaño de los circulos indica cantidad de elementos por comunidad.

Tabla 3. Comunidades Bibliográficas (Tendencias) más relevantes en Iberoamerica.

\begin{tabular}{|c|c|c|c|}
\hline Número & Comunidad & $\begin{array}{l}\text { Palabras Clave (10) } \\
\text { en orden de relevancia }\end{array}$ & $\begin{array}{c}\text { Países de investigación (3) en } \\
\text { orden de relevancia }\end{array}$ \\
\hline 1 & $\begin{array}{l}\text { Instrumentos } \\
\text { psicométricos-Depresión }\end{array}$ & $\begin{array}{l}\text { Depresión, niños, adolescentes, escala, stress, propieda- } \\
\text { des psicométricas, comportamiento, salud, validación, } \\
\text { cuestionario,sintomas. }\end{array}$ & España, Chile, Portugal \\
\hline 2 & Demencia-Alzheimer & $\begin{array}{l}\text { Demencia, Enfermedad de Alzheimer, depresión, desempeño, } \\
\text { diagnóstico, dificultad, prevalencia, mini-mental, déficit, escala. }\end{array}$ & España, Brasil, Argentina \\
\hline 3 & $\begin{array}{c}\text { Instrumentos } \\
\text { psicométricos-Poblaciones }\end{array}$ & $\begin{array}{l}\text { Psicología, Guía, validez, validación, adolescentes, preva- } \\
\text { lencia, sintomas, mujeres, desordenes, salud. }\end{array}$ & España, México, Portugal \\
\hline 4 & Prevalencia & $\begin{array}{l}\text { Prevalencia, depresión, mujeres, síntomas, desórdenes de } \\
\text { ansiedad, calidad de vida, anorexia nerviosa, comorbilidad, } \\
\text { factores de riesgo, esquizofrenia. }\end{array}$ & España, Brasil, Portugal \\
\hline 5 & $\begin{array}{l}\text { Instrumentos } \\
\text { Psicométricos-Patologías }\end{array}$ & $\begin{array}{l}\text { Validación, propiedades psicométricas, esquizofrenia, des- } \\
\text { orden, síntomas, depresión, ansiedad, anorexia nerviosa, } \\
\text { fobia, clasificación. }\end{array}$ & España, Brasil, Portugal \\
\hline 6 & Psicoterapia & $\begin{array}{l}\text { Depresión, psicoterapia, comportamiento, asimiliación, } \\
\text { inventario, terapia, mujeres, yo, alianza de trabajo, anorexia } \\
\text { nerviosa. }\end{array}$ & España, Chile, Portugal \\
\hline 7 & Desórdenes alimenticios & $\begin{array}{l}\text { Anorexia nerviosa, mujeres, bulimia nerviosa, desordenes } \\
\text { alimenticios, prevalencia, síntomas, validación, actitudes, } \\
\text { tempreamento, desórdenes. }\end{array}$ & España, Portugal, Chile \\
\hline 8 & Psicofármacos & $\begin{array}{l}\text { Depresión, Ensayo controlado al azar, olanzapina, doble- } \\
\text { ciego, seguimiento, asociaciones, esquizofrenia, eficacia, } \\
\text { aceptación, síntomas. }\end{array}$ & España, Brasil, Portugal \\
\hline 9 & Salud Mental & $\begin{array}{l}\text { Depresión, Prevalencia, Salud mental, Encuesta nacional } \\
\text { de comorbilidad, cuestionario, Estados Unidos, Depresión, } \\
\text { Desórdenes, Modelo, Impacto, OMS. }\end{array}$ & España, Mexico, Colombia \\
\hline 10 & Abuso Sustancias & $\begin{array}{l}\text { Prevalencia, Abuso, Alcohol, Experiencias adversas en la } \\
\text { niñez, drogodependiencia, dependencia, acceso privilegiado, } \\
\text { adicción, fumar, disfunción familiar. }\end{array}$ & Brasil, Argentina, Mexico \\
\hline
\end{tabular}


psicométricos como elemento de estudio en las investigaciones abarcan la mayor cantidad de categorías, pudiendo diferenciarlas según el uso que se les da a estos o los objetivos que se buscan alcanzar, en relación a estudios de diferentes poblaciones o patologías.

Destaca el estudio de la depresión a nivel transversal, el Alzheimer y desórdenes alimenticios como patologías más relevantes de estudio, en tanto comprenden categorías propias.

También es posible apreciar como objeto de estudio la psicoterapia y la salud mental en un rol central, en relación a estrategias de intervención e importancia de factores como la alianza o el cambio terapéutico.

\section{Discusión}

La producción iberoamericana ha crecido de manera constante durante los últimos años. Es posible apreciar que en este grupo la mayor cantidad de publicaciones se concentran en Europa (España y Portugal), encontrándose en Latinoamérica el $37 \%$ de las investigaciones, donde Brasil, Chile y México lideran la producción en Iberoamérica con $27 \%$.

Aunque la posición de Chile pueda parecer sorprendente, debido a una tamaño mucho menor de su población en comparación con Brasil y México, es coherente con estudios que predicen una relación entre PIB per cápita, inversión en I+D y producción científica (De Moya-Anegon y HerreroSolana, 1999; Lortie, 2009). Actualmente Chile tiene un PIB per cápita superior tanto a Brasil como a México, aunque una inversión en I+D como porcentaje del PIB menor (The World Bank, 2013).

De manera coherente, las investigaciones en psicología clínica aparecen ligadas a factores económicos tanto a nivel general como en inversión en la ciencia de cada país más que a la población específica del mismo, mostrando la importancia de la generación de recursos para la investigación en ciencias sociales, necesitando también de las gestiones estatales y la generación de iniciativas para poder surgir, al igual que otras áreas científicas.

Sin embargo, pese al crecimiento de las publicaciones y la presencia de estos países en la producción científica se aprecia una influencia casi absoluta de España y Portugal en cuanto al liderazgo de las publicaciones. Si bien en el conteo total la ciencia latinoamericana aporta con más de un tercio de la producción, en el análisis de las publicaciones más influyentes en el periodo, la presencia latinoamericana se reduce al 13\%. Excluido Brasil, el conteo de liderazgo latinoamericano entre los más citados se reduce al 3\% mostrando que a pesar del número de publicaciones existen desafíos significativos en cuanto a calidad y liderazgo para los científicos latinoamericanos.

En psicología es posible identificar esta tendencia de agrupación de países a la hora de generar investigación, donde se encuentran exponentes líderes, dentro de los cuales sólo España aparece como representante de habla hispana a nivel mundial, estableciéndose en Estados Unidos el foco más importante de la investigación en psicología clínica y de la salud (Adair y Huynh, 2012) lo que se corrobora con los resultados obtenidos y da cuenta de la importancia de la globalización y la internacionalización en investigación psicológica. Asimismo, el uso del idioma inglés como lengua predominante de los autores iberoamericanos es coherente con la aceptación de este idioma como lengua franca, y que a su vez está relacionado con más citas, mayor factor de impacto y accesibilidad a una audiencia mucho mayor (Vinther y Rosenberg, 2012).

Con respecto a los tipos de investigación analizadas aparece un claro predominio de los estudios cuantitativos, principalmente en relación a instrumentos psicométricos, tanto en la creación como validación de los mismos, además del uso dentro de la investigación para medición de las variables. El énfasis en psicometría en psicología clínica desarrolla un fuerte cimiento para luego, en las investigaciones futuras poder contar con un cuerpo de instrumentos apropiados para generar conocimiento en líneas correlaciónales o incluso explicativas de ciertas conductas, trastornos o eventos, haciendo que se robustezca el análisis obtenido y la aplicación de los mismos en los campos de acción de la disciplina.

Esto da cuenta de la evolución de la psicología clínica, al menos en el ámbito de la investigación, en la búsqueda de medición cuantitativa de las variables que se estudian, en la creación de instrumentos y en la importancia de la validación de los cuestionarios que se utilizan, adaptándose a las diferentes poblaciones que se estudian aceptando que, si bien la tendencia en investigación es a la globalidad, dentro de cada comunidad se hace necesaria la mirada específica en relación a aspectos sociales y culturales.

Dicho esto, también existe una hegemonía de referencias metodológicas y un escaso número de citas referidas a orientaciones terapéuticas especifica. Esto es coherente con lo observado por otros estudios (Heatherington, Messer, Angus, Strauman, Friedlander y Kolden, 2013) que describen una reducción de las visiones teóricas enseñadas en los programas de doctorado en psicología clínica. Otro análisis de las orientaciones de estos programas (Levy y Anderson, 2013) también revela un declive de todas las orientaciones teóricas excepto la cognitiva conductual. 
Los autores creen que estos análisis muestran indicios de ser coherentes con estas tendencias internacionales. Las hipótesis de este fenómeno son variadas y sobrepasan los límites del presente estudio, pero presentan preguntas interesantes en torno a la formación de los investigadores, y como se desarrolla y fomenta la investigación de diversas corrientes teóricas en la psicología clínica.

En cuanto a los temas más estudiados, se centran en torno a la depresión, ligando las investigaciones al área psiquiátrica en relación a la influencia de fármacos en el tratamiento de diferentes trastornos, comorbilidad y procesos cognitivos asociados a las patologías, observándose una alianza con el área médica-psiquiatrica más que con otras disciplinas de las ciencias sociales. Destaca la incipiente asociación con Neurociencias, que aparece como la novena subdisciplina Web of Knowledge con mayor asociación a la psicología clínica (Tabla 1) lo que invita a pensar en nuevas formas, multidisciplinarias, de enfrentar los desafíos de la disciplina.

La depresión aparece como el trastorno más frecuente, afectando a más de 350 millones de personas, como tal es la principal causa de incapacidad a nivel mundial (American Psychological Association, 2013; World Health Organization, 2013a), aparenciendo entonces coherente la importancia que se le da a este trastorno en las investigaciones iberoamericanas.

Tambíen aparece el Alzheimer dentro de las comunidades de análisis más estudiadas, lo que ligado a la aparición de neurociencias, gerontología y estudio de la familia como categorías con mayor aparición da cuenta que la salud mental en el adulto mayor es otro de los intereses actuales de la psicología clínica.

Dado el aumento en la esperanza de vida y la tendencia al envejecimiento que se observa en las poblaciones de los países estudiados (World Health Organization, 2013b; Kinsella y He, 2009), es posible proyectar esta misma tendencia de publicaciones para los años futuros, destacando la relevancia de la psicología en las políticas públicas, orientando las preguntas de investigación a los problemas que se viven en la población (Fischhoff, 1990).

A nivel general, los estudios se centran por una parte en la enfermedad y por otra en los aspectos biológicos y farmacológicos en relación a tratamiento, más que en elementos psicoterapéuticos u otras aproximaciones. Esto contrasta con la perspectiva de salud mental de la Organización Mundial de la Salud (2013), referida a un estado de bienestar, donde el individuo es consciente de sus capacidades, puede enfrentar las situaciones estresantes propias de la vida, trabajar y ser un aporte a la comunidad. Esto se enmarca en la definición de salud general que realiza la misma institución, centrada en el bienestar más que en la ausencia de la patología (Park, Peterson y Sun, 2013).

En relación a la predominancia de estudios biológicos y farmacológicos, aquellos que se relacionan con procesos cognitivos pueden tener alguna aplicación más concreta por ejemplo en la psicoterapia, sin embargo, dentro de estos artículos no se aprecian elementos que permitan por ejemplo distinguirlos de la psiquiatría, lo que coincide con la clasificación de los mismos artículos y las tendencias de investigación ya comentadas. Los estudios que se relacionan con procesos cognitivos pueden tener alguna aplicación más concreta por ejemplo en las intervenciones psicoterapéuticas, sin embargo dentro de estos artículos no se aprecian elementos que permitan por ejemplo distinguirlos de la psiquiatría, lo que coincide con la clasificación de los mismos artículos.

Dentro de la investigación en psicoterapia propiamente tal destacan como elemento central los elementos que se traducen en el exito de la intervención, vale decir indicadores, significancia, factores comunes y cambio terapéuticco, mostrando el interés de la investigación por generar conocimiento que permita desarrollar y fortalecer técnicas y herramientas que estén orientadas a la eficacia del proceso, más que las caraterísticas del mismo, o el estudio del paciente.

En cuanto a las limitaciones de este tipo de estudios, la definición de Psicología Clínica, aunque se ajusta a un criterio internacional como la categorización Web of Knowledge-ISI, es susceptible de ser mejorada y ampliada, incorporando tanto nuevos conceptos, así como nuevas bases de datos tales como SCOPUS. Es importante hacer notar que la metodología de áreas de la ciencia (WC) incorpora revistas de otras disciplinas como la psiquiatría, neurología y gerontología, por lo que se entiende la psicología clínica como un área temática amplia y no limitada por la profesión o estudios de los autores.

Como reflexión final, es necesario resaltar que este tipo de análisis es particularmente relevante para investigadores y practicantes, ya que permiten analizar con respecto al rol de la psicología a largo plazo así como ponderar adecuadamente los juicios de expertos sobre el actual estado y el futuro de la psicología clínica. Estos modelos también pueden ser utilizados para explorar la relación de la Psicología con otras ciencias y conocer las temáticas que se estudian en las fronteras de la misma.

También permite ponderar la situación de la ciencia Ibero y Latinoamericana. Históricamente, el peso de la ciencia producida en latinoamericana es menor al $2 \%$ de la ciencia mundial (De Moya-Anegon y Herrero-Solana, 1999). Nuestro análisis revela que para el periodo 2012, esta alcanza el 4\%. El haber duplicado el peso relativo, liderados por Brasil, 
refleja los esfuerzos de la región por producir ciencia de nivel mundial aunque deja pendientes algunos desafíos, como el del bajo liderazgo de las publicaciones de mayor impacto o la menor cantidad de citas que reciben los papers de autores latinoamericanos (Menenghini, Packer y Nassi-Calo, 2008). En este contexto paree particularmente importante la necesidad de que la ciencia en Ibero y Latinoamérica evite este sesgo y pondere de manera objetiva las contribuciones científicas de sus pares en esta región del mundo.

En cuanto a los desafíos de la psicología clínica ibero y latinoamericana, la búsqueda de mayor influencia, líneas teóricas propias, mayor énfasis en el bienestar por sobre las enfermedades o el desarrollo de instrumentos que agreguen valor por sobre la validación de instrumentos bien pueden ser objetivos estratégicos de unidades o grupos de investigación. La hegemonía del inglés y la influencia casi unidireccional de los países desarrollados a Latinoamérica obliga a pensar la mejor manera de generar ciencia de nivel mundial pensando en audiencias globales pero orientadas a responder de la mejor manera a las comunidades locales.

\section{Referencias}

Adair, J. G., y Huynh, C. L. (2012). Internationalization of psychological research: Publications and collaborations of the United States and other leading countries. International Perspectives in Psychology: Research, Practice, Consultation, 1, 252-267.

American Psychological Association. (2013). Depression. Recuperado desde $h$ ttp://apa.org/topics/depress/index.aspx

Amézquita, J., Martínez, D., Martínez, J. C., y Maza, F. (2011). Bibliometría, Infometria y Cienciometría. Cartagena: Ediciones Unicartagena.

Ariza, T., y Reina Granados, M. (2012). Bibliometric Analysis of the most relevant Iberoamerican Journals related to Clinical and Health Psychology of the Journal Citation Reports (2011). Terapia Psicológica, 30, 89-102.

Barrios, M., Vilarroya, A., y Borrego, A. (2013). Scientific production in psychology: a gender analysis. Scientometrics, 95, 15-23.

De Moya-Anegon, F., y Herrero-Solana, V. (1999). Science in America Latina: A comparison of Bibliometric and scientific-technical indicators. SCIENTOMETRICS , 46, 299-320.

Duffy, R., Jadidian, A., Webster, G., y Sandell, K. (2011). The research productivity of academic psychologists: assessment, trends, and best practice recommendations. Scientometrics, 89, 207-227.

Fischhoff, B. (1990). Psychology and Public Policy. American Psychologist, 45, 647-653.

Fleischhacker, W., Cetkovich-Bakmas, M., De Hert, M., Hennekens, C., Lambert, M., Leucht, S. (2008). Comorbid somatic illnesses in patients with severe mental disorders: Clinical, policy, and research challenges. Journal of Clinical Psychiatry, 69, 514-519.

Grauwin, S., Beslon, G., Fleury, E., Franceschelli, S., Roberdet, C., .. Rouquier, J. (2012). Complex Systems Science: Dreams of Universality,
Reality of Interdisciplinarity. Journal of the American Society for Information Science and Technology, 63, 1327-1338. doi: 10.1002/asi.22644.

Grawin, S., y Jensen, P. (2011). Mapping Scientific Institutions. Scientometrics, 89, 943-954. doi: 10.1007/s11192-011-0482-y.

Heatherington, L., Messer, S., Angus, L., Strauman, T., Friedlander, M., y Kolden, G. (2013). The narrowing of Theoretical orientations in Clinical Psychology Doctoral Training. Clinical Psychology Science and Practice, 19, 364-374.

Kinsella, K. y He, W. (2009) An aging world 2008: International population reports. US. Department of Health and Human Services. Recuperado desde http://www.census.gov/prod/2009pubs/p95-09-1.pdf

Levy, K., y Anderson, T. (2013). Is Clinical Psychology Doctoral Training Becoming Less Intellectually Diverse? And If So, What Can Be Done? Clinical Psychology-Science and Practice, 20, 211-220.

Lortie, C. (2009). Letter to the Editor: A global comment on scientific publications, productivity, people and beer. Scientometrics, 84, 539-541. doi: 10.1007/s11192-009-0077-z.

Menenghini, R., Packer, A., y Nassi-Calo, L. (2008). Articles by Latin American Authors in Prestigious Journals Have Fewer Citations. PLOS ONE, 3, 1-8. doi: 10.1371/journal.pone.0003804.

Moya-Anegón, F., Guerrero-Bote, V., Bornmann, L., y Moed, H. (2013). The research guarantors of scientific papers and the output counting: a promising new approach. Scientometrics, 97, 421-434. doi: 10.1007/ s11192-013-1046-0.

Navarrete-Cortes, J., Fernandez-Lopez, J., Lopez-Baena, A., QuevedoBlasco, R., y Buela-Casal, G. (2010). Global Psychology: A bibliometric Analysis of Web of Science Publications. Universitas Psychologica, 9, 553-567.

Olese, P., y von Ins, M. (2010). The rate of growth in scientific publication and the decline in coverage provided by Science Citation Index. Scientometrics, 84, 575-603. doi: 10.1007/s11192-010-0202-z.

Organización Mundial de la Salud. (2013). Salud Mental: un estado de bienestar. Recuperado desde http://www.who.int/features/factfiles/ mental health/es/index.html

Park, N., Peterson, C., y Sun, J. (2013). La Psicología Positiva: Investigación y aplicaciones. Terapia Psicológica, 31, 11-19.

Quevedo-Blasco, R., y Lopez-Lopez, W. (2011). Status of iberomerican journals of psychology at the 2010 Journal Citation Reports. Universitas Psychologica, 10, 937-947.

SCIMago Group (2013). SJR Global Rank. Recuperado desde http://www. scimagoir.com/pdf/SIR\%20Global\%202013\%20O.pdf

Reuters, T. (2013). www.wok.com. Recuperado desde: http://wokinfo. com/citationconnection/

The World Bank. (2013). Indicators. Recuperado desde http://data.worldbank.org/indicator/NY.GDP.PCAP.CD

Vera-Villarroel, P., López-López, W., Lillo, S., y Silva, L. (2011). La producción científica en psicología Latinoamericana: un análisis de la investigación por países. Revista Latinoamericana de Psicología, 43, 95-104

Vinther, S., y Rosenberg, J. (2012). Impact factor trends for general medical journals: non-English-language journals are lagging behind. Swiss Medical Weekly, 142, 1-6.

World Health Organization. (2013a). Depression. Recuperado desde http:// www.who.int/mediacentre/factsheets/fs369/en/index.html

World Health Organization. (2013b). Life expectancy at birth. Recuperado desde http://gamapserver.who.int/gho/interactive charts/mbd/ life_expectancy/atlas.html

Zych, I., y Buela-Casal, G. (2010). Internationality of the Multidisciplinary Psychology Journals Published in Ibero-America and Included in the Web Of Science. Universitas Psychologica, 9, 27-34. 
